\title{
AN ELEMENTARY PROOF OF THE TRACZYK-TOKOTA CRITERIA FOR PERIODIC KNOTS
}

\author{
JÓZEF H. PRZYTYCKI
}

(Communicated by Ronald Stern)

\begin{abstract}
Traczyk used the first coefficient of the skein (Homfly) polynomial to find powerful criteria for $r$ periodic knots. The criteria were extended by Yokota to $(r-1) / 2$ first coefficients of the skein polynomial. We give here a short, elementary proof of the Traczyk-Yokota criteria. The main tool is the Jaeger composition product, the same product which is a base for Turaev's Hopf algebra structure of links in a handlebody.
\end{abstract}

An oriented link $L$ in $S^{3}$ is said to be $r$-period ( $r$ an odd, prime number) if it has a diagram which is invariant under the rotation, $\varphi$, of $R^{2}$ by a $\frac{2 \pi}{r}$ angle. Let $l k(L, \gamma)$ denote the linking number of $L$ with the rotation axis, $\gamma$.

The skein polynomial of oriented links, $P_{L} \in Z\left[v^{ \pm 1}, z^{ \pm 1}\right]$, is characterized by the properties: $P_{T_{n}}=\left(\frac{v^{-1}-v}{z}\right)^{n-1}$, where $T_{n}$ is the trivial link of $n$ components, and $v^{-1} P_{L_{+}}-v P_{L_{-}}=z P_{L_{0}}$, where $L_{+}, L_{-}$, and $L_{0}$ make up the standard skein triple. If $K$ is a knot, then $P_{K}=\sum_{i=0} P_{2 i}(K) z^{2 i}$, where $P_{2 i}(K) \in Z\left[v^{ \pm 2}\right]$.

Traczyk [4] and Yokota [5] proved the following property of the skein polynomial of periodic knots:

Theorem 1. Let $K$ be an r-periodic knot and $k=l k(L, \gamma)$. Then:

(a) (Traczyk) If $P_{0}(K)=\sum a_{2 i} v^{2 i}$ then $a_{2 i} \equiv a_{2 i+2} \bmod r$ except possibly when $2 i+1 \equiv \pm k \bmod r$.

(b) (Yokota) $P_{2 i}(K) \equiv b_{2 i} P_{0}(K) \bmod r$ for $2 i<r-1$, where numbers $b_{2 i}$ depend only on $r$ and on $k \bmod r$.

Notice that because $K$ is a knot, therefore $k$ is coprime with $r$. The crucial part of the proof, by Traczyk and Yokota, is an involved analysis of the skein polynomial of torus knots (using the Jones formula [2]). In our proof we do not need a reduction to torus knots at all and instead use the Jaeger composition product formula [1] (we should stress that Jaeger proved his formula by elementary considerations involving Reidemeister moves).

It is convenient to work with the following regular isotopy variant of the

Received by the editors August 4, 1993.

1991 Mathematics Subject Classification. Primary 57M25.

Key words and phrases. Knot, skein polynomial, Homfly, periodic knot, Jaeger composition product. 
skein polynomial:

$$
Q_{D}(v, z)=z^{\operatorname{com}(D)-1} v^{-\operatorname{Tait}(D)}\left(v^{-1}-v\right) P_{D}(v, z)
$$

where $\operatorname{com}(D)$ is the number of link components and Tait $(D)$ is the algebraic sum of the signs of the crossings of $D$. It is also convenient to add the empty link, $\varnothing$, to the set of links and put $Q_{\varnothing}(v, z)=1 . Q_{D}(v, z)$ satisfies the skein relation

$$
Q_{D_{+}}-Q_{D_{-}}= \begin{cases}Q_{D_{0}} & \text { in the case of a selfcrossing } \\ z^{2} Q_{D_{0}} & \text { in the case of a mixed crossing }\end{cases}
$$

and $Q_{D \cup 0}=\left(v^{-1}-v\right) Q_{D}$. The advantage of working with $Q_{D}(v, z)$ is that $Q_{D}(v, z) \in Z\left[v^{ \pm 1}, z^{2}\right]$ (no negative powers of $z$ ) and that the Jaeger composition product has a nice simple form. We will write now the formula and its reduction modulo $\left(r, z^{r-1}\right)$ in the case of $r$-periodic knots.

Theorem 2 (Jaeger [1]). Let $D$ be a diagram of an oriented link in $S^{3}$. Then:

$$
Q_{D}\left(v_{1} v_{2}, z\right)=\sum_{f \in l b l(D)}\langle D \mid f\rangle v_{2}^{\operatorname{rot}\left(D_{f, 1}\right)} Q_{D_{f, 1}}\left(v_{1}, z\right) v_{1}^{--\operatorname{rot}\left(D_{f, 2}\right)} Q_{D_{f, 2}}\left(v_{2}, z\right)
$$

where the meaning of the used symbols is as follows: To define $l b l(D)$, consider $D$ as a 4-valent graph. Let $E d g(D)$ denote the set of the graph $D$. By a 2labelling of $D$ we mean a function $f: \operatorname{Edg}(D) \rightarrow\{1,2\}$ such that around $a$ vertex the following labellings are allowed:

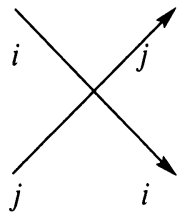

(possibly $i=j$ )
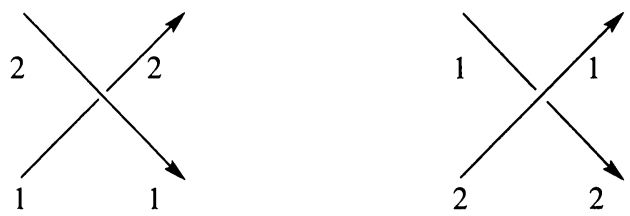

The set of 2-labellings of $D$ is denoted by $\operatorname{lbl}(D)$. The edges of $D$ with label $i$ form an oriented link diagram denoted by $D_{f, i}$. The vertices of $D$ which are neither in $D_{f, 1}$ nor $D_{f, 2}$ are called $f$-smoothing vertices of $D$. Let $|f|_{-}$(resp., $|f|_{+}$) denote the number of negative (resp., positive) $f$-smoothing vertices of $D$. Let $|f|=\left|f_{-}\right|+|f|_{+}$and $\langle D \mid f\rangle=(-1)^{|f|} \mid-z^{|f|-\operatorname{com}\left(D_{f, 1}\right)-\operatorname{com}\left(D_{f, 2}\right)+\operatorname{com}(D)}$. Finally let $\operatorname{rot}(D)$ be the rotational number of $D$, i.e., $\operatorname{rot}(D)$ is the sum of the signs of the Seifert circles of $D$ where the sign of such a circle is 1 if it is oriented counterclockwise and -1 otherwise.

Corollary 3. Let $D$ be an r-periodic knot diagram in $R^{2}$. Then Jaeger's composition product reduces to

$$
\begin{aligned}
Q_{D}\left(v_{1} v_{2}, z\right) & \equiv \sum_{f \in l b l_{s p e c}(D)}(-1)^{|f|-v_{2}^{r o t}\left(D_{f, 1}\right)} \\
& \times Q_{D_{f, 1}}\left(v_{1}, z\right) v_{1}^{-\operatorname{rot}\left(D_{f, 2}\right)} Q_{D_{f, 2}}\left(v_{2}, z\right) \bmod \left(r, z^{r-1}\right)
\end{aligned}
$$

where $f \in \operatorname{lbl}_{\text {spec }}(D)$ if $f \in \operatorname{lbl}(D)$ and the following conditions hold:

(a) $f$ is a $\varphi$-invariant labelling,

(b) exactly one component of $D_{f, 1} \cup D_{f, 2}$ is $\varphi$ invariant (other components are permuted by $\varphi$ ). 
Proof. (a) $\varphi$ acts on the set of labellings and orbits of the action have $r$ elements or one $\varphi$-invariant labelling. Elements of the same orbit introduce the same to the formula, so modulo $r$ we are left with $\varphi$-invariant labellings.

(b) Let $f$ be a $\varphi$-invariant labelling. $\varphi$ acts on the set of components of the link diagrams $D_{f, 1}, D_{f, 2}$ and orbits of this action have one or $r$ elements. Let $C$ be the number of 1-element orbits. Then $|f|-\operatorname{com}\left(D_{f, 1}\right)-\operatorname{com}\left(D_{f, 2}\right)+$ $\operatorname{com}(D) \geq(r-1)(C-1)$; in particular, if $C>1$ then we can ignore $f$ in the sum considered modulo $z^{r-1}$. To prove the above inequality let us consider a graph, $G$, whose vertices are components of $D_{f, 1} \cup D_{f, 2}$ and edges correspond to $f$-smoothed crossings. $\varphi$ acts on $G$ with the quotient $G_{*}$. Furthermore the action on edges of $G$ is free (so orbits have $r$ elements) and the $\varphi$ action on vertices has $C$ fixed points. Because $D$ is a knot diagram, $\operatorname{com}(D)=1$ and both $G$ and $G_{*}$ are connected. In particular $E\left(G_{*}\right) \geq V\left(G_{*}\right)-1$. Comparing the number of edges and vertices in $G$ and $G_{*}$, one gets: $|f|=E(G)=$ $r E\left(G_{*}\right) \geq r V\left(G_{*}\right)-r=V(G)+r C-C-r=\operatorname{com}\left(D_{f, 1}\right)+\operatorname{com}\left(D_{f, 2}\right)+$ $(r-1)(C-1)-1$. This completes the proof of $(b)$.

Corollary 4. Let $D$ be an r-periodic knot diagram, $f \in l b l_{\text {spec }} D$ and $K_{f}$ be the unique $\varphi$-invariant component of $D_{f, 1} \cup D_{f, 2}$ (see Corollary 3(b)). Let lbl $l_{\text {spec }}^{(\mathrm{i})}$ be the subset of $l b l_{s p e c} D$ such that $K_{f}$ is in $D_{f, i}$ and $f$ is not constant. Then

$$
\begin{aligned}
Q_{D}\left(v_{1} v_{2}, z\right) \equiv & v_{2}^{r o t D} Q_{D}\left(v_{1}, z\right)+v_{1}^{-r o t D} Q_{D}\left(v_{2}, z\right) \\
& +\sum_{f \in l b l_{\text {spec }}^{(1)}} v_{2}^{k} Q_{K_{f}}\left(v_{1}, z\right)\left(v_{2}^{-1}-v_{2}\right)^{r} Q^{(1)}\left(v_{1}^{r}, v_{2}^{r}\right) \\
& +\sum_{f \in l b l_{\text {spec }}^{(2)}} v_{1}^{-k} Q_{K_{f}}\left(v_{2}, z\right)\left(v_{1}^{-1}-v_{1}\right)^{r} Q^{(2)}\left(v_{1}^{r}, v_{2}^{r}\right) \bmod \left(r, z^{r-1}\right),
\end{aligned}
$$

where $Q^{(1)}\left(v_{1}, v_{2}\right), Q^{(2)}\left(v_{1}, v_{2}\right) \in Z\left[v_{1}^{ \pm 1}, v_{2}^{ \pm 1}\right]$.

Proof. Assume that $f \in l b l_{s p e c}^{(1)}$. Let $D_{f}^{(1)}$ be a sublink of $D_{f, 1}-K_{f}$ which contains exactly one component from each orbit of the $\varphi$ action on components of $D_{f, 1}-K_{f}$. Similarly let $D_{f}^{(2)}$ be a sublink of $D_{f, 2}$ which contains exactly one component from each $\varphi$ orbit. Then, as noted in $[4,5]$,

$$
\begin{gathered}
Q_{D_{f, 1}}(v, z) \equiv\left(v^{-1}-v\right)^{r}\left(\frac{Q_{D_{f}^{(1)}}(v, z)}{v^{-1}-v}\right)^{r} Q_{K_{f}}(v, z) \bmod \left(r, z^{2 r-2}\right), \\
Q_{D_{f, 2}}(v, z) \equiv\left(v^{-1}-v\right)^{r}\left(\frac{Q_{D_{f}^{(2)}}(v, z)}{v^{-1}-v}\right)^{r} \bmod \left(r, z^{2 r-2}\right) .
\end{gathered}
$$

Similar formulas hold in the case of $f \in l b l_{\text {spec }}^{(2)}$.

To finish the proof of Corollary 4 , note that for any $r$-periodic link diagram $L$ one has $\operatorname{rot}(L) \equiv l k(L, \gamma) \bmod r$ where $\gamma$ is the axis of the rotation.

Before we prove Theorem 1 we formulate first some elementary properties of polynomials which we use when applying the Jaeger composition product:

Lemma 5. Let $R$ denote a commutative ring with 1 , let $Q(v)$ be an $R$ Laurent polynomial in variable $v$, and let $s$ be an integer coprime with $r$ : 
(a) If

$$
Q\left(v_{1} v_{2}\right) \equiv v_{2}^{s} Q\left(v_{1}\right)+v_{1}^{-s} Q\left(v_{2}\right) \bmod \mathscr{I}
$$

where $\mathscr{I}=\{0\}$ or $\left(v_{1}^{2 r}-1, v_{2}^{2 r}-1\right)$, then $Q(v) \equiv c\left(v^{-s}-v^{s}\right) \bmod \mathscr{I}^{\prime}$ for some $c \in R$, where $\mathscr{I}^{\prime}=\{0\}$ or $\left(v^{2 r}-1\right)$.

(b) If

$$
Q\left(v_{1} v_{2}\right)=v_{2}^{s} Q_{1}\left(v_{1}, v_{2}^{2 r}\right)+v_{1}^{-s} Q_{2}\left(v_{1}^{2 r}, v_{2}\right)
$$

where $Q_{1}, Q_{2} \in R\left[v_{1}^{ \pm 1}, v_{2}^{ \pm 1}\right]$, then $Q(v)=v^{s} Q^{\prime}\left(v^{2 r}\right)+v^{-s} Q^{\prime \prime}\left(v^{2 r}\right)$ for some $Q^{\prime}(v), Q^{\prime \prime}(v) \in R\left[v^{ \pm 1}\right]$.

(c) If (b) holds and additionally $Q_{1}\left(v_{1}, 1\right)=Q\left(v_{1}\right)$ and $Q_{2}\left(1, v_{2}\right)=Q\left(v_{2}\right)$, then $Q(v)=\left(v^{-s}-v^{s}\right) Q^{\prime \prime \prime}\left(v^{2 r}\right)+\left(v^{s}-v^{-s+2 r}\right) Q^{(i v)}\left(v^{2 r}\right)$ for some $Q^{\prime \prime \prime}(v)$, $Q^{(i v)}(v) \in R\left[v^{ \pm 1}\right]$.

Proof. Let $Q(v)=\sum c_{i} v^{i}$.

(a) The only possible nonzero terms are $c_{s} v^{s}$ and $c_{-s} v^{-s}$. Furthermore $c_{s}+c_{-s}=0$, so we can put $c=c_{-s} . Q(v)$ for which Lemma 5(a) holds is said to be primitive modulo $\mathscr{I}^{\prime}$.

(b) We compare terms on the left and right sides of the equation. On the left side we have only terms with the same exponent of $v_{1}$ as that of $v_{2}$, so the only monomials on the right side which can survive are of the form $v_{2}^{s+2 r t} v_{1}^{s+2 r t}$ and $v_{1}^{-s+2 r t^{\prime}} v_{2}^{-s+2 r t^{\prime}}$, which completes the proof of Lemma 5(b).

(c) This follows because $Q\left(v_{1} v_{2}\right)$ satisfies (a) when considered modulo $\left(v_{1}^{2 r}-1, v_{2}^{2 r}-1\right)$ and thus $Q(v) \equiv c\left(v^{-s}-v^{s}\right) \bmod \left(v^{2 r}-1\right)$.

Lemma 6. For $s$ coprime with $r$, there is the unique polynomial $c_{r, s} \in Z\left[z^{2}\right]$ of $z$-degree at most $r-3$, such that for $z=v^{-1}-v: c_{r, s}\left(v^{-s}-v^{s}\right) \equiv$ $\left(v^{-1}-v\right) v^{r(s-1)} \bmod \left(v^{2 r}-1\right)$ in $Z\left[v^{ \pm 1}\right]$. Furthermore $c_{r, s}=c_{r, s+r}=-c_{r,-s}$ and $c_{r, s}(0) s \equiv 1 \bmod r$.

Proof. One can easily check, using the Euclidean algorithm, that $\frac{v^{-s}-v^{s}}{v^{-1}-v} v^{-r(s-1)}$ is invertible in $Z\left[\left(v^{-1}-v\right)^{2}\right] /\left(\frac{v^{-r}-v^{r}}{v^{-1}-v}\right)$. Let $\sum_{i=0}^{(r-3) / 2} b_{2 i}^{\prime}\left(v^{-1}-v\right)^{2 i}$ be the unique polynomial of degree at most $r-3$ in variable $\left(v^{-1}-v\right)$ representing this inverse. Then $c_{r, s}$ from Lemma 6 is equal to $\sum_{i=0}^{(r-3) / 2} b_{2 i}^{\prime} z^{2 i}$. For $v=1$, so $z=0$, one gets $b_{0}^{\prime} s \equiv 1 \bmod r$.

Lemma 7. Let $Q(v, z) \in Z\left[z^{2}\right]\left[v^{ \pm 1}\right]$ be a polynomial of $z$-degree at most $r-3$ which satisfies the following two properties:

(a) For $z=v^{-1}-v$, one has $Q(v, z) \equiv\left(v^{-1}-v\right) v^{r(s-1)} \bmod \left(v^{2 r}-1\right)$.

(b) $Q\left(v_{1} v_{2}, z\right)=v_{2}^{s} Q\left(v_{1}, z\right)+v_{1}^{-s} Q\left(v_{2}, z\right)+c_{r, s} W\left(v_{1}, v_{2}\right)$, where $W\left(v_{1}, v_{2}\right) \in Z\left[v_{1}^{ \pm 1}, v_{2}^{ \pm 1}\right]$ and $W\left(v_{1}, v_{2}\right) \equiv 0 \bmod \left(v_{1}^{2 r}-1, v_{2}^{2 r}-1\right)$.

Then $Q(v, z)=c_{r, s} W^{\prime}(v)$ for some polynomial $W^{\prime}(v) \in Z\left[v^{ \pm 1}\right]$.

Proof. Let $Q(v, z)=\sum v^{i} c_{i}(z)$ and $W\left(v_{1}, v_{2}\right)=\sum b_{i, j} v_{1}^{i} v_{2}^{j}$. By comparing the coefficients of $v_{1}^{i} v_{2}^{j}$ in the formula (b) one gets: $c_{i}(z)=-c_{r, s} b_{i, s}=$ $-c_{r, s} b_{-s, i}=c_{r, s} b_{i, i}$ if $i \neq \pm s, c_{s}(z)+c_{-s}(z)=-b_{-s, s} c_{r, s}$, and $b_{i, j}=0$ if $i \neq j, i \neq-s$, and $j \neq s$; finally $b_{s, s}=b_{-s,-s}=0$. The polynomial $Q(v, z)$ is primitive modulo $\left(v^{2 r}-1\right)$; therefore, by Lemma $5, Q(v, z) \equiv$ $c\left(v^{-s}-v^{s}\right) \bmod \left(v^{2 r}-1\right)$. Then by assumption (a) and Lemma 6 one has $c=c_{r, s}$. On the other hand $c=\sum_{j} c_{-s+2 j r}(z)=-\sum_{j} c_{s+2 j r}(z)$; therefore, because $s \not \equiv-s \bmod 2 r$, the conclusion of Lemma 7 follows. 
Now we can deduce the Traczyk-Yokota criteria from Corollary 4 by applying Lemmas 5-7.

(1) $Q_{D}(v, z)$ satisfies the assumptions of Lemma 7 modulo $\left(r, z^{r-1}\right)$, for $s=\operatorname{rot}(D)$; therefore, Theorem $1(\mathrm{~b})$ holds with $b_{2 i} \equiv b_{2 i}^{\prime} s \bmod r$. Namely: For any link diagram $L, P_{L}\left(v, v^{-1}-v\right)=1$, so for a $\operatorname{knot}$ diagram $D$, one has $Q_{D}\left(v, v^{-1}-v\right)=\left(v^{-1}-v\right) v^{-T a i t(D)} \equiv\left(v^{-1}-v\right) v^{r(s-1)} \bmod \left(v^{2 r}-\right.$ 1). We use the general fact that $\operatorname{rot}(L) \equiv \operatorname{Tait}(L)+\operatorname{com}(L) \bmod 2$. Thus $Q_{D}(v, z) \bmod \left(r, z^{r-1}\right)$ satisfies (a) of Lemma 7 for $s=\operatorname{rot}(D)$. It also satisfies (b), and the proof is by induction on the number of crossings, $\operatorname{cr}(D)$, of $D$. For $D$ such that $l b l_{\text {spec }}(D)$ contains only constant functions (e.g., $\operatorname{cr}(D)=0$ ), it follows immediately from Corollary 4 . An inductive step also follows immediately from Corollary 4; one has only to observe that if $f$ is not constant, then $K_{f}$ has less crossings than $D$ and one can apply to $K_{f}$ the inductive assumption (one has to notice that $\operatorname{rot}(D) \equiv \operatorname{rot}\left(K_{f}\right) \equiv k \bmod r$ and that $c_{r, s}=c_{r, s+r}$ ).

(2) $Q_{D}(v, z)$ satisfies the assumptions of Lemma 5(c) modulo $\left(r, z^{r-1}\right)$; therefore,

$$
Q_{D}(v, z) \equiv\left(v^{-s}-v^{s}\right) Q^{\prime \prime \prime}\left(v^{2 r}, z\right)+\left(v^{s}-v^{-s+2 r}\right) Q^{(i v)}\left(v^{2 r}, z\right) \bmod \left(r, z^{r-1}\right)
$$

for some $Q^{\prime \prime \prime}(v, z), Q^{(i v)}(v, z) \in Z\left[v^{ \pm 1}, z\right]$. Namely: as in case (1) we perform an induction on $\operatorname{cr}(D)$, starting from the case when $l b l_{\text {spec }}(D)$ contains only constant functions. The crucial observation here is that $v^{s} Q_{D}(v, z)$ has only even powers of $v$; therefore, we can perform an inductive step using Corollary 4. Now

$$
\begin{aligned}
& P_{D}(v, z)=\frac{1}{v^{-1}-v} v^{\operatorname{Tait}(D)} Q_{D}(v, z) \\
& \equiv v^{\text {Tait }(D)}\left(\left(v^{-s+1}+v^{-s+3}+\cdots+v^{s-3}+v^{s-1}\right) Q^{\prime \prime \prime}\left(v^{2 r}, z\right)\right. \\
& \left.\quad+\left(v^{s+1}+v^{s+3}+\cdots+v^{-s+2 r-3}+v^{-s+2 r-1}\right) Q^{(i v)}\left(v^{2 r}, z\right)\right) \bmod \left(r, z^{r-1}\right) .
\end{aligned}
$$

Therefore, Theorem 1(a) holds and the proof of the Traczyk-Yokota theorem is complete.

Our method extends to $r^{i}$-periodic knots. Furthermore, using Jaeger's skein state model for the Kauffman polynomial, it extends to periodicity criteria yielded by the Kauffman polynomial. We hope to describe these in the future paper.

\section{REFERENCES}

1. F. Jaeger, Composition products and models for the Homfly polynomial, Enseign. Math. 35 (1989), 323-361.

2. V. Jones, Hecke algebra representations of braid groups and link polynomials, Ann. of Math. (2) 126 (1987), 335-388.

3. K. Murasugi, Jones polynomials of periodic links, Pacific J. Math. 131 (1988), 319-329.

4. P. Traczyk, Periodic knots and the skein polynomial, Invent. Math. 106 (1991), 73-84.

5. Y. Yokota, The skein polynomial of periodic knots, Math. Ann. 291 (1991), 281-291.

Department of Mathematics and Computer Science, Odense University DK-5230, ODENSE M, DENMARK

Current address: Department of Mathematics, University of California, Berkeley, California 94720

E-mail address: Jozef@math.berkeley.edu 